\title{
Frecuencia de indiferencia al tratamiento odontológico en adultos de 18 a 44 años, Totoracocha, Cuenca-Ecuador, 2017
}

\author{
Frequency of indiference to the dental treatment in adults from 18 to 44 years old \\ Totoracocha, Cuenca-Ecuador, 2017
}

María José Fernández Siguencia ${ }^{1 \mathrm{a}}$, Liliana Encalada Verdugo ${ }^{1 \mathrm{~b}}$, Mayra Alexandra Siguencia Gavilánez $^{2 \mathrm{c}}$

\section{RESUMEN}

Objetivo: Determinar la frecuencia de indiferencia al tratamiento odontológico en adultos jóvenes de 18 a 44 años, de la parroquia Totoracocha, Cuenca-Ecuador, 2017. Materiales y Métodos: Se realizó un estudio cuantitativo con diseño descriptivo transversal en individuos de ambos sexos, comprendidos entre las edades de 18 a 44 años a quienes se les aplicó la encuesta DIS de Nuttall, el tamaño de la muestra se calculó con la fórmula de muestreo probabilístico, estratificado y proporcional, más un $10 \%$ por efectos de pérdida encuestando a 586 personas. La información se ingresó a la base de datos EPI Info 7, descartando las encuestas que no presentaban consentimiento informado, que tenían tachones, y grupos etarios no pertenecientes a la edad correspondiente, la información fue revisada por el área de investigación de la carrera de odontología. Respecto a los aspectos éticos la investigación fue aprobada por el comité de ética de la Universidad Católica de Cuenca, Facultad de Odontología. Resultados: La frecuencia de indiferencia al tratamiento odontológico que se presentó en la parroquia Totoracocha fue del $76 \%$ de la población encuestada, donde la afección a los hombres fue $39 \%$ y en mujeres fue $37 \%$, la causa principal de indiferencia fue la falta de tiempo con un porcentaje de $23 \%$. Conclusión: Más del $50 \%$ de encuestados presentaron indiferencia al tratamiento odontológico donde el factor más importante fue la falta de tiempo.

Palabras claves: Apatía; prevalencia; Adultos jóvenes; Consulta odontológica; Actitud. (Fuente: DeCS BIREME)

\section{ABSTRACT}

Objective: To determine the frequency of indifference to dental treatment in young adults aged 18 to 44 , from the Totoracocha parish, Cuenca-Ecuador, 2017. Materials and Methods: A quantitative study with a descriptive crosssectional design was carried out in individuals of both sexes. , between the ages of 18 to 44 years to whom the Nuttall DIS survey was applied, the sample size was calculated with the probabilistic, stratified and proportional sampling formula, plus $10 \%$ for loss effects, surveying 586 persons. The information was entered into the EPI Info 7 database, discarding the surveys that did not present informed consent, that had studs, and age groups not belonging to the corresponding age, the information was reviewed by the research area of the dental career. Regarding ethical aspects, the research was approved by the ethics committee of the Catholic University of Cuenca, Faculty of Dentistry. Results: The frequency of indifference to the dental treatment that was presented in the Totoracocha parish was $76 . \%$ of the population surveyed, where the condition for men was 39\% and for women it was 37\%, the main cause Indifference was the lack of time with a percentage of $23 \%$. Conclusion: More than $50 \%$ of respondents presented indifference to dental treatment where the most important factor was the lack of time.

Key words: Apathy; prevalence; Young adults; Dental consultation; Attitude. (Source: MeSH NLM)

${ }^{1}$ Universidad Católica de Cuenca, carrera de Odontología.

Cuenca, Ecuador.

${ }^{2}$ Consejo de la Judicatura. Loja, Ecuador.

${ }^{\text {a Odontóloga }}$

${ }^{\mathrm{b}}$ Docente

c Psicólogo, Perito

Correspondencia:

María José Fernández Siguencia

Dirección: Cuenca-Ecuador. Teléfono: 0983039081

Correoelectrónico: siguenciafernandez.majo@hotmail.com
Este es un artículo Open Access distribuido bajo la licencia Creative Commons Atribución-No ComercialCompartir Igual 4.0

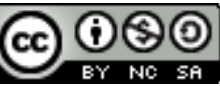

Citar como: Fernandez Siguencia MJ, Encalada Verdugo L, Siguencia Gavilanez MA. Frecuencia de indiferencia al tratamiento odontológico en adultos de 18 a 44 años, Totoracocha, Cuenca, Ecuador 2017. KIRU. 2020;17(3):117-122. https://doi.org/10.24265/kiru.2020.v17n3.01 


\section{INTRODUCCIÓN}

La indiferencia es una condición afectiva neutra, en la cual el individuo se encuentra al margen de un problema o situación, es importante indagar y establecer las causas de esta actitud, la familia es un pilar fundamental para la superación de este inconveniente, aunque también suele requerirse ayuda profesional psicológica ${ }^{(1-4)}$.

La indiferencia al tratamiento odontológico consiste en acciones negativas realizadas por la población, definiéndola como la insensibilidad, la falta de motivación para resolver problemas bucodentales, tomándoles a estos como algo secundario sin mayor importancia, dando como resultado secuelas incómodas para el individuo ${ }^{(1,2,4)}$.

La indiferencia al cuidado dental se mide mediante el cuestionario desarrollado por Nuttall en el año 1996, la cual consta de 8 preguntas, estas tienen la finalidad de identificar y medir actitudes relacionadas con la salud bucodental, este se caracteriza por ser rápido, corto y de fácil aplicación ${ }^{(3-6)}$.

Se consideran factores asociados a la indiferencia al tratamiento odontológico, la edad (ya que aquí el individuo adquiere responsabilidades tales como una pareja, ser padres), un título universitario y un trabajo estable ${ }^{(2,5,6)}$. El sexo juega un papel importante, por su naturaleza la mujer se preocupa más por la parte física y estética, mientras que el hombre no se guía por su apariencia ni por el buen estado de salud ${ }^{(5,7)}$, (en ellos prima, el ser proveedores del hogar), la instrucción académica no influye en el acceso a los servicios odontológicos, la economía juega un papel importante dado que las personas con mayor nivel socioeconómico acuden más al dentista debido a que tienen mayor información, motivación y dinero ${ }^{(5-9)}$. La salud bucodental debería ser una prioridad al igual que la general, la actitud indiferente puede ser causante de alteraciones como: gingivitis, periodontitis, y lesiones cariosas ${ }^{(10-12)}$.

En la terapéutica para la indiferencia es importante motivar a la persona, mediante el adiestramiento en medidas preventivas como técnicas de higiene, uso de hilo dental, enjuague bucal y visitas periódicas al odontólogo; así también explicar de manera simple y entendible el diagnóstico de las enfermedades presentes y las opciones de tratamientos $^{(13-17)}$.

Estudios realizados anteriormente como el de Skaret (18), en el año 2010 denominado "Confiabilidad y validez de la escala de indiferencia dental en una población de jóvenes de 18 años en Noruega", indica que la prevalencia de indiferencia se presentó en más del $50 \%$ de la población masculina, Marshman Zoe ${ }^{(5)}$, en Inglaterra en el año 2014, en una muestra de reclusos, reporta la existencia de indiferencia dental en un $94.7 \%$, sin embargo la información acerca de prevalencia de la indiferencia al tratamiento odontológico en la edad de 18 a 44 años sigue siendo limitada a nivel de Ecuador y Sudamérica.

El propósito del estudio es identificar la frecuencia de la indiferencia al tratamiento odontológico en adultos de 18 a 44 años, de la parroquia Totoracocha, así como los factores asociados con la indiferencia al tratamiento odontológico.

\section{MATERIALES Y MÉTODOS}

Se realizó un estudio cuantitativo con diseño descriptivo transversal, en hombres y mujeres, entre de 18 a 44 años de edad, de la parroquia Totoracocha, (En Ecuador se denomina Parroquia a la división político-territorial de menor rango) esta investigación pertenece al macro proyecto denominado "Barreras para el acceso al servicio Odontológico".

Los participantes a quienes se les aplicó la encuesta de DIS Nuttall, la cual mide la indiferencia a los tratamientos odontológicos y consta de 8 preguntas cada una representa un punto dando un total de 8 puntos, un puntaje de $0-1$ expresa no indiferencia y de 2 a 8 indica indiferencia al tratamiento odontológico, el plan de análisis corresponde a determinar la frecuencia de indiferencia, se aplicó tipo de muestreo probabilístico, estratificado y proporcional, el tamaño de la muestra se calculó con la fórmula para población finita, con un nivel de confiabilidad de $95 \%$ y un error de $5 \%$ siendo la población de la parroquia Totoracocha de 25,430 habitantes de 0 a 80 años según los datos de INEN 2010 (Instituto Ecuatoriano de Normalización), cuyo instituto informa sobre el número de personas pertenecientes a cada, provincia, ciudad, y parroquia, la información que proporciona no incluye datos personales, siendo así la muestra de 379 encuestados, más un $10 \%$ por efectos de pérdida, encuestando a 586 personas, cuyo estrato para el muestreo es el grupo de edad, sin importar el sexo.

Por ser el estudio parte de un macro proyecto, primero se calibró al investigador con un experto para aplicar la encuesta, las visitas fueron realizadas los fines de semana, durante ocho horas diarias, el acceso a los 
los entrevistados fue mediante búsqueda de puerta a puerta, se explicó a los participantes sobre la investigación, y el desarrollo del cuestionario de Nutall, el cual fue validado al idioma español por el área de investigación de la Universidad Católica de Cuenca.

Se solicitó el consentimiento informado, en el cual se garantiza que el encuestado ha expresado voluntariamente su intención de participar en el estudio, y posteriormente ser beneficiados con la información, y recomendaciones que pueda arrojar los resultados del estudio.

Se excluyeron los datos erróneos, personas con discapacidades físicas o mentales y edades que no correspondan al grupo etario, ya que las encuestas se aplicaron a todas las edades, ya que la investigación pertenece a un macro proyecto, la obtención de información errónea fue producto de la aceleración de los encuestados en terminar la encuesta, y la exclusión de dichas fichas fue por parte del área de investigación de la Universidad Católica de Cuenca.
Entre las limitaciones que se encontró fueron la movilización debido a la extensión de la parroquia, el horario, ya que las encuestas únicamente se las realizaba el fin de semana y la colaboración de la población. Una vez recolectada la muestra y verificados los datos estos fueron ingresados al programa EPI INFO versión 7. De libre acceso, se revisó la veracidad de la información para la validación interna, luego esta base de datos fue revisada por el Departamento de Investigación donde se obtuvo la validación externa, y con ello el control de calidad, luego de esto se ejecutó la tabulación y el análisis de variables cualitativas por medio de la prueba chi-cuadrado.

\section{RESULTADOS}

La indiferencia al tratamiento odontológico se presentó en un $76 \%$ de la población. (tabla 1). En relación al sexo, la indiferencia se presentó de manera uniforme (tabla 2). De acuerdo al grupo etario, la indiferencia se presentó con mayor frecuencia en el grupo de 18-27 años, siendo significativa en relación a los demás grupos (tabla 3).

Tabla 1. Frecuencia de la indiferencia al tratamiento odontológico en adultos jóvenes de 18 a 44 años, de la parroquia Totoracocha, Cuenca-Ecuador, 2017

\begin{tabular}{lcc}
\hline Indiferencia & $\mathbf{n}$ & $\mathbf{\%}$ \\
\hline Indiferente & 446 & 76 \\
No indiferente & 140 & 24 \\
Total & 586 & 100 \\
\hline
\end{tabular}

Tabla 2. Frecuencia de la indiferencia al tratamiento odontológico, en personas de la parroquia Totoracocha según sexo.

\begin{tabular}{lcccccc}
\hline Sexo & Indiferente & \multicolumn{3}{c}{ No Indiferente } & \multicolumn{3}{c}{ Total } \\
& $\mathbf{n}$ & $\mathbf{\%}$ & $\mathbf{n}$ & $\mathbf{\%}$ & $\mathbf{n}$ & $\mathbf{\%}$ \\
\hline Femenino & 214 & 37 & 76 & 13 & 290 & 50 \\
Masculino & 231 & 39 & 65 & 11 & 296 & 50 \\
Total & 445 & 76 & 141 & 24 & 586 & 100 \\
\hline
\end{tabular}

Tabla 3. Frecuencia de indiferencia al tratamiento odontológico, en personas de 18 a 44 años de la parroquia Totoracocha, según grupo etario.

\begin{tabular}{lcccccc}
\hline Edad & Indiferente & \multicolumn{2}{c}{ No Indiferente } & \multicolumn{2}{c}{ Total } \\
& $\mathbf{n}$ & $\mathbf{\%}$ & $\mathbf{n}$ & $\mathbf{\%}$ & $\mathbf{n}$ & $\%$ \\
\hline 18-27años & 308 & 53 & 97 & 16 & 405 & 69 \\
28-37 años & 94 & 16 & 27 & 5 & 121 & 21 \\
38-44 años & 43 & 7 & 17 & 3 & 60 & 10 \\
Total & 445 & 76 & 141 & 24 & 586 & 100 \\
\hline
\end{tabular}


Entre los factores asociados que provocan Indiferencia se puntualiza con un mayor porcentaje, falta de tiempo y tratamiento doloroso (tabla 4). Con relación al nivel de instrucción, se halló mayor indiferencia al tratamiento odontológico, en encuestados con educación superior universitaria seguido de los que tenían educación secundaria (tabla 5).

Tabla 4. Indiferencia al tratamiento odontológico, en personas de 18 a 44 años de la parroquia Totoracocha, según los factores asociados para la indiferencia al tratamiento odontológico.

\begin{tabular}{lcc}
\hline Factores asociados & $\mathrm{n}$ & $\%$ \\
\hline Tratamiento doloroso. & 131 & 22.3 \\
Demasiado tiempo. & 95 & 16.2 \\
Preocupación o ansiedad. & 61 & 11.0 \\
Culpa por el estado dental. & 30 & 4.5 \\
Cuesta mucho. & 87 & 14.9 \\
Falta de tiempo. & 134 & 23.0 \\
Pospongo mis citas. & 48 & 8.1 \\
& & \\
Total: & 586 & $100 \%$ \\
\hline
\end{tabular}

Tabla 5. Indiferencia al tratamiento odontológico, en personas de 18 a 44 años de la parroquia Totoracocha, según el nivel de instrucción.

\begin{tabular}{|c|c|c|c|c|c|c|}
\hline \multirow[t]{2}{*}{ Instrucción } & \multicolumn{2}{|c|}{ Indiferente } & \multicolumn{2}{|c|}{ No Indiferente } & \multicolumn{2}{|c|}{ Total } \\
\hline & $\mathbf{n}$ & $\%$ & $\mathbf{n}$ & $\%$ & $\mathbf{n}$ & $\%$ \\
\hline Analfabeto & 2 & 0.2 & 0 & 0 & 2 & 0.2 \\
\hline Primaria & 17 & 2.9 & 2 & 0.4 & 19 & 3.3 \\
\hline Secundaria & 169 & 28.8 & 51 & 8.7 & 105 & 37.5 \\
\hline $\begin{array}{l}\text { Superior técnica } \\
\text { Superior }\end{array}$ & 76 & 13.9 & 23 & 3.2 & 51 & 17.1 \\
\hline Universitaria & 181 & 30.4 & 65 & 11.5 & 246 & 41.9 \\
\hline Total & 445 & $76.2 \%$ & 141 & $23.8 \%$ & 586 & $100 \%$ \\
\hline
\end{tabular}

\section{DISCUSIÓN}

Los resultados del estudio indicaron que existe una alta frecuencia de indiferencia al tratamiento odontológico en el $76 \%$ de la población estudiada, de acuerdo al grupo etario los resultados indicaron que la indiferencia estuvo porcentualmente marcada en la edad de $18-27$ años, con respecto al sexo tanto masculino como femenino presentan frecuencias similares con un $39 \%$ y $37 \%$ respectivamente.

Erick Skaret, en el año 2010 en un estudio realizado en Noruega reporta que la indiferencia estuvo presente en más del $50 \%$ de la población masculina, mientras que en el presente estudio se reporta una indiferencia del $76 \%$ en la población, posiblemente porque los datos reflejan información del sexo masculino y femenino ${ }^{(18)}$.

Marshman Zoe, en Inglaterra en el año 2014, realizó un estudio en la penitenciaría donde encuestó a 659 reclusos de sexo masculino, reportando que existe indiferencia al cuidado dental en un $94.7 \%$ estos resultados indican un porcentaje elevado similar al obtenido en esta investigación, esto puede deberse a que al estar 
recluidos la presencia de enfermedades bucodentales está ligada a la falta de servicios de salud que se dispone únicamente en casos de emergencia ${ }^{(5)}$.

En el presente estudio se reporta, dos principales factores asociados a la indiferencia al tratamiento odontológico, que son la falta de tiempo y el tratamiento doloroso en un $23 \%$ y $22.3 \%$ respectivamente, causas que estuvieron presentes en un alto porcentaje para ambos sexos y en el rango etario de 18 a 27 años, esto puede deberse a que por la falta de tiempo las personas priorizan otras actividades, dejando a lado su salud bucal, además el hecho de realizarse un tratamiento odontológico desde la antigüedad ha sido denominado como doloroso, sin embargo en el estudio realizado por Nuttall (3), en el año 1996, en Escocia, en el cual validó varias preguntas para determinar la presencia de indiferencia al tratamiento dental, no se analiza la información de acuerdo a los factores asociados para la existencia de indiferencia.

De acuerdo al nivel de instrucción y la indiferencia, los resultados de esta investigación se encuentra porcentualmente superiores en personas de instrucción alta en un $2 \%$ más que el nivel bajo de instrucción, siendo este dato similar para ambos sexos.

Nigel Nuttall, en el año 1996, en su estudio realizado en Escocia, relaciona la indiferencia con la ocupación y reporta que los obreros presentan un $53 \%$ de esta, si relacionamos con la variable nivel de instrucción de este estudio podríamos asociar que los obreros pertenecen al nivel bajo de instrucción según Nuttall presentan menor indiferencia porcentual que los de ocupación profesional ${ }^{(3)}$.

Con los resultados obtenidos del estudio se concluye que la indiferencia al tratamiento odontológico afecta a una gran parte de las personas, existiendo varios factores, siendo la falta de tiempo el principal, es importante recalcar que el tratamiento odontológico desde la prehistoria ha sido temido sobre todo por el dolor que genera. La indiferencia es un tema no muy estudiado a nivel Mundial, nacional, regional y local por lo que es importante profundizar el tema con nuevas investigaciones para así poder extrapolar los resultados.

Contribución de autoría: MJFS, participó en el diseño del estudio, recolección y análisis de datos. LEV y MASG participaron en la obtención de datos y la redacción del manuscrito. Todos los autores aprobaron el artículo.
Fuente de financiamiento: Ninguna.

Conflictos de interés: Los autores no presentaron conflictos de interés.

\section{REFERENCIAS BIBLIOGRÁFICAS}

1. García M. Las consecuencias de la indiferencia. La mente es maravillosa. 2014.

2. Piqueras J. Emociones negativas y salud. Researchgate. 2010; 18(1):85-112.

3. Nuttall NM; Initial development of a. scale to measure dental indifference. Community Dent Oral Epidemiol 1996; 24; 112-6.

4. Lopez A. Montiel J, Almerich J. Estudio de la satisfacción del paciente en odontología mediante cuestionarios de salud: adaptación al español del cuestionario "dental satisfaction questionnaire”. Roderic. 2012. Disponible en: http://roderic.uv.es/handle/10550/24925

5. Marshman Z, Baker SR, Robinson PG. Does dental indifference influence the oral healthrelated quality of life of prisoners? Community Dent Oral Epidemiol 2014; 42: 470-480.

6. Jamieson LM, Thomson WM. The Dental Neglect and Dental Indifference scales compared. Community Dent Oral Epidemiol 2002; 30: 168-75.

7. González S. Epidemiología de la caries dental en la población venezolana. Revista de ciencias médicas. La Habana. 2014; 20(2):208218.

8. Carvajal Paola. Enfermedades periodontales como un problema de salud pública: el desafío del nivel primario de atención en salud. Rev. Clin. Periodoncia Implantol. Rehabil. Oral [Internet]. $2016 \quad$ Ago [citado 2020 Mar 3] ; 9( 2 ): 177-183. Disponible en: https://scielo.conicyt.cl/scielo .php?script=sci_arttext\&pid=S0719-010720160 00200016\&lng =es

9. Velasco I. Principios para el tratamiento de infecciones odontogénicas con distintos niveles de complejidad. Revista chilena de cirugía. 2012; 64(6):586-598.

10. Álvarez J. Actualización de aspectos relacionados con el Síndrome del Diente Fisurado. Revista Habanera de Ciencias Médicas. 2015 abril; 14(4):397-408.

11. Mateo-Sidrón Antón M.C., Somacarrera Pérez M.L.. Cáncer oral: genética, prevención, diagnóstico y tratamiento. revisión de la literatura. Av Odontoestomatol [Internet]. 2015 Ago [citado 2020 feb 10] ; 31( 4 ): 247259. Disponible en: http://scielo.iscii.es/ /scielo.php?script=sci_arttext\&pid=S0213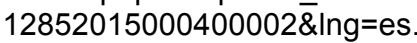

12. Reyes BH, Gómez, Hidalgo SH, Nodarse RL. Bruxismo: panorámica actual. Scielo.2017;21(1).

13. Rojas Alcayaga A, Misrachi Laurent C. Avances en odontoestomatología. 2004;20(4):185-191. 
14. Navarro C. Un estudio epidemiológico acerca de la prevalencia de ansiedad y miedos dentales entre la población adulta de la gran área metropolitana de costa rica. Psicología conductual. 1996; 4(1). Disponible http://www.funveca.org/revista/PDFespanol/ 1996/art05.1.04.pdf

15. Maurer R. El método Kaisen, Un pequeño paso puede cambiar tu vida (Crecimiento personal). Barcelona; 2015.

16. Dilla T. Adherencia y persistencia terapéutica: causas, consecuencias y estrategias de mejora. ELSEVIER. 2009 Junio; 41(6); 295-354.

17. Acosta M, Debs G, Noval R, Dueñas A. Conocimientos, creencias y prácticas en pacientes hipertensos, relacionados con su adherencia terapéutica. Rev Cub Enferm. 2005 Septiembre- Diciembre; 21(3).

18. Skaret E, Raadal M, Kvale G, Berg E. Reliability and validity of the Dental Indifference Scale in a population of 18year-olds in Norway. Community Dent Oral Epidemiol 2000; 28:330-5.

\author{
María José Fernández Siguencia \\ Siguenciafernandez.majo@hotmail.com \\ Liliana Soledad Encalada Verdugo \\ lencalada@ucacue.edu.ec \\ Mayra Alexandra Siguencia Gavilánez \\ masiguenciag@hotmail.com
}

(D) https://orcid.org/0000-0001-7555-7143

(D) https://orcid.org/0000-0001-8804-1138

(C) https://orcid.org/0000-0002-0586-9181 